\title{
Surface Potential of Polycrystalline Hematite in Aqueous Medium
}

\author{
Tajana Preočanin, Filip Stipić, Atiđa Selmani, and Nikola Kallay \\ Laboratory of Physical Chemistry, Department of Chemistry, Faculty of Science, University of Zagreb, Horvatovac 102A, \\ 10000 Zagreb, Croatia \\ Correspondence should be addressed to Tajana Preočanin, tajana@chem.pmf.hr
}

Received 30 April 2011; Accepted 14 June 2011

Academic Editor: José H. Zagal

Copyright ( $\odot 2011$ Tajana Preočanin et al. This is an open access article distributed under the Creative Commons Attribution License, which permits unrestricted use, distribution, and reproduction in any medium, provided the original work is properly cited.

\begin{abstract}
The surface potential of polycrystalline hematite in aqueous sodium perchlorate environment as a function of $\mathrm{pH}$ was examined. Surface potential of hematite was obtained from measured electrode potential of a nonporous polycrystalline hematite electrode. Acidic solution was titrated with base, and the backward titration with acid was performed. Substantial hysteresis was obtained which enabled location of the point of zero potential and equilibrium values of surface potentials. The theoretical interpretation of the equilibrium data was performed by applying the surface complexation model and the thermodynamic equilibrium constants for the first and the second step of surface protonation was obtained as $\log K_{1}^{\circ}=11.3 ; \log K_{2}^{\circ}=2.8$.
\end{abstract}

\section{Introduction}

Interfacial layers in heterogeneous systems exhibit special properties. Important systems are minerals in aqueous medium. Ionic species from aqueous solutions interact with surface sites resulting in surface charge. Binding and release of charged species from/of the solid surface is markedly affected by the electrostatic potential at the interface; that is, ionic equilibrium within the interfacial layer is influenced by the electrostatic potential. On the other hand, this potential is the result of the accumulation of charged ionic species at the interface. Accordingly, surface potential is one of the main parameters characterizing equilibrium within the ionic interfacial layer dividing two phases. For minerals in the aqueous environment, surface charge and potential is the result of interactions with aqueous $\mathrm{H}^{+}$and $\mathrm{OH}^{-}$ions so that these ions are considered as potential determining ions, p.d.i. Consequently, surface charge and potential is a function of $\mathrm{pH}$. To evaluate surface potential one may use two routes. The first one is indirect. The surface charge density is measured by potentiometric acid-base titrations [1] and surface complexation model (SCM) [2-4] is applied. The second one is the direct measurements of surface potential, either by constructing a corresponding electrode $[5,6]$ or by applying the ion sensitive field effect transistors (ISFETs) $[7,8]$. There were several attempts to measure the surface potential. The first one was based on the platinum wire covered with hematite particles [9]. However, due to the porosity of the metal oxide layer, the electrode behaved as the electrode of the second kind, the potential of which is determined by the redox equilibrium at the metal surface and the solubility of the metal oxide. More recently, the problem of porosity was solved by construction of the single crystal metal oxide electrodes [5]. This approach enabled measurements of the surface potential of hematite $[5,6,10-13]$, titania $[14,15]$, pyrite [16], sapphire [17], ice [18, 19], and silver halides [20-23]. The advantage of single crystal (SCr) electrodes is that it is possible to measure properties of individual and specified crystal plane $[6,15,24,25]$. To examine properties of colloid suspensions, for example, colloid stability due to electrostatic repulsion between particles, it is necessary to be aware that several crystal planes are exposed to the aqueous environment. This paper reports results obtained with polycrystalline ( $\mathrm{PCr}$ ) hematite electrode, made from the natural hematite being compact and nonporous agglomerate of small hematite particles.

\section{Experimental and Results}

All chemicals used in this study were of the analytical grade purity. Hematite used for the construction of the nonporous polycrystalline electrode was a natural sample composed of 


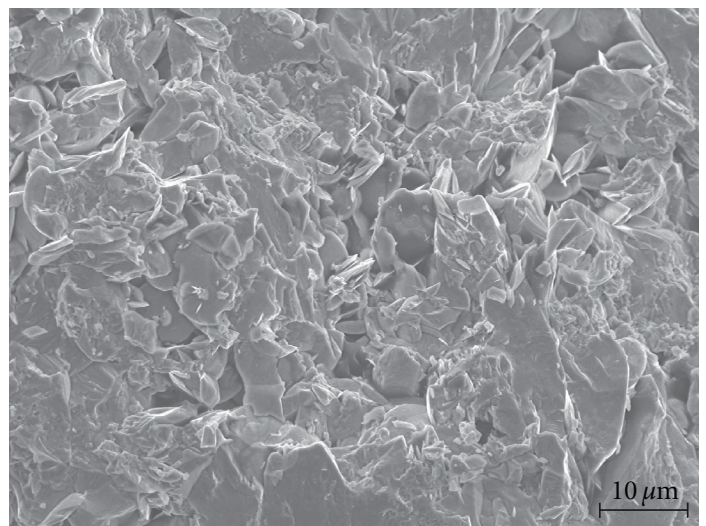

FIgure 1: Polycrystalline hematite.

hematite platelets as shown in Figure 1. The packing is dense with void volume fraction of about $5 \%$. The absence of filling the pores by water was tested by weighing the original sample and storing it in water overnight. The mass was the same so that it was concluded that water had not filled the pores of the polycrystalline hematite.

The nonporous polycrystalline hematite electrode was constructed as shown in Figure 2. Polycrystalline hematite was connected to the copper wire with conductive glue and all contacts were isolated with plexiglas.

The electrode potential $E$ of the nonporous polycrystalline hematite electrode was measured with respect to the reference silver chloride electrode $\left(c_{\mathrm{KCl}}=3 \mathrm{~mol} \mathrm{dm}^{-3}\right)$ accompanied by the salt bridge filled with a solution of the same content as the initial measuring solution (Metrohm 6.0729.100.). The resistance of the polycrystalline hematite electrode was measured as $1.5 \mathrm{G} \Omega$ so that Methrom $713 \mathrm{pH}$ meter had enough high impedance to ensure proper readings. $\mathrm{pH}$ was measured using glass electrode (Metrohm 6.0133.100.) and the same reference silver chloride electrode as for the polycrystalline hematite electrode. Separate $\mathrm{pH}$ meter operated by batteries (Methrom 826) was used for measurements of $\mathrm{pH}$ values so that mutual influence through grounding was excluded. Systems were thermostated at $25.0^{\circ} \mathrm{C}$. Experiments were performed under an argon atmosphere, to avoid $\mathrm{CO}_{2}$ contamination. The entire measuring system (thermostated vessel, electrodes, magnetic stirrer, and dosimate) was placed in Faraday cage so that external electrical effects were avoided. Polycrystalline hematite electrode was cleaned with ethanol and rinsed with water before the measurements.

Titrations were performed as follows: $200 \mathrm{~cm}^{3}$ of aqueous solution of perchloric acid $\left(c=10^{-3} \mathrm{~mol} \mathrm{dm}{ }^{-3}\right)$ was titrated with $0.1 \mathrm{~mol} \mathrm{dm}{ }^{-3} \mathrm{NaOH}$ solution. The readings were taken 10 minutes after each addition of the titrant. For backward titration, $0.1 \mathrm{~mol} \mathrm{dm}^{-3} \mathrm{HClO}_{4}$ solution was used. Again, the readings were taken 10 minutes after addition of the titrant. The results are presented in Figure 3. The observed hysteresis was attributed to slow equilibration at the hematite surface, being substantially faster in acidic $(\mathrm{pH} \approx 3)$ and in basic $(\mathrm{pH} \approx 10)$ environment than in the neutral $\mathrm{pH}$ region.

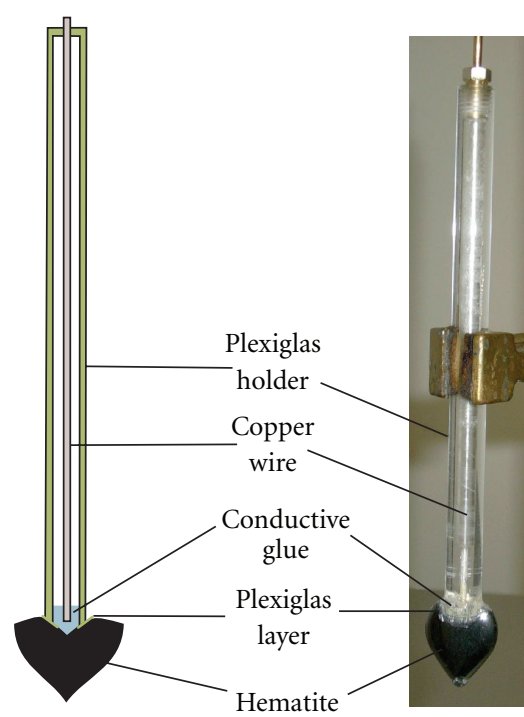

FIGURE 2: Nonporous polycrystalline hematite electrode.

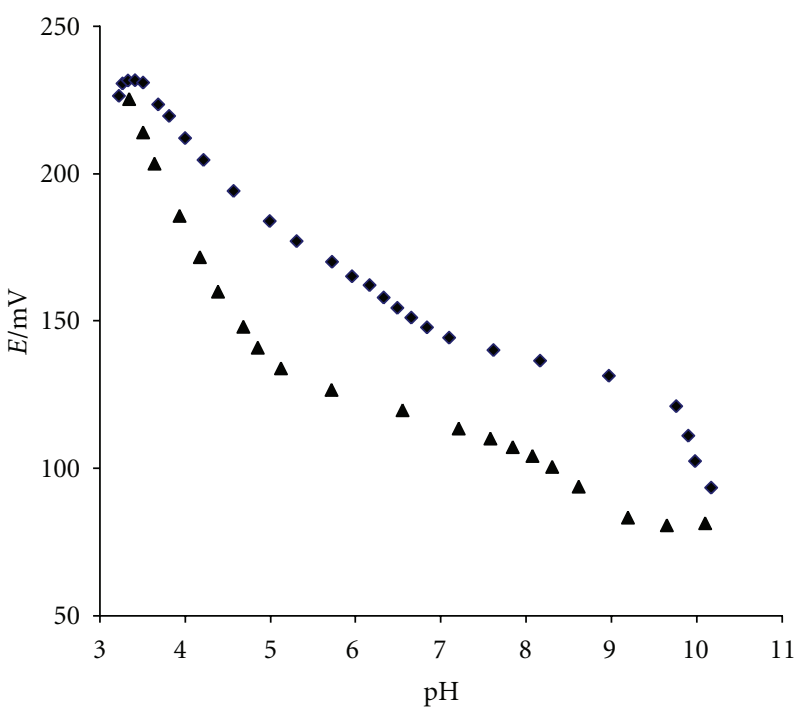

FIGURE 3: Electrode potential of nonporous polycrystalline hematite electrode (equilibration time: $\sim 10 \mathrm{~min}$ ) as a function of $\mathrm{pH}$ of the solution in the presence of $\mathrm{NaClO}_{4}\left(c=10^{-3} \mathrm{~mol} \mathrm{dm}{ }^{-3}\right)$. Titration of acidic solution with $\mathrm{NaOH}(\checkmark)$, and back titration of basic solution with $\mathrm{HClO}_{4}(\boldsymbol{\Delta})$. Temperature: $25.0^{\circ} \mathrm{C}$.

The ultrasound was applied to promote equilibration at the hematite surface. Forward and backward titrations were performed in the same way as described in the previous paragraph. However, after each addition of the titrant the system was treated with ultrasound probe $(220 \mathrm{~W})$ for $\sim 1 \mathrm{~min}$ and left to equilibrate for $\sim 10 \mathrm{~min}$. Results are presented in Figure 4.

\section{Evaluation of Data}

The measurements shown in Figures 3 and 4 are of the open circuit type. The obtained electrode potential $E$ is in 


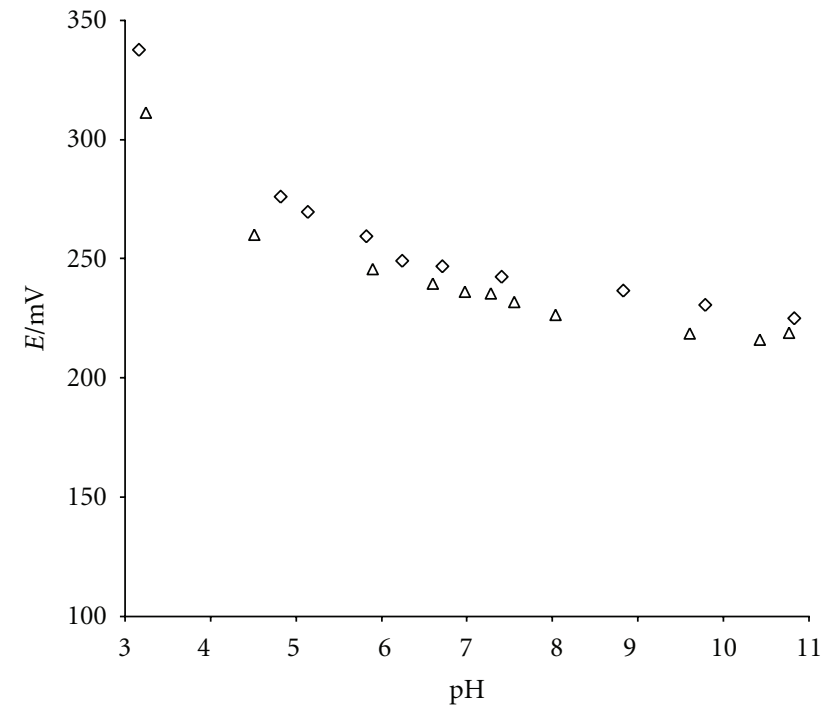

FIGURE 4: Electrode potential of the nonporous polycrystalline hematite electrode as a function of $\mathrm{pH}$ of the solution in the presence of $\mathrm{NaClO}_{4}\left(c=10^{-3} \mathrm{~mol} \mathrm{dm}{ }^{-3}\right)$. Titrations were performed under the ultrasound, using the ultrasound probe $(220 \mathrm{~W})$. Titration of acidic solution with $\mathrm{NaOH}(\diamond)$ and back titration of basic solution with $\mathrm{HClO}_{4}(\triangle)$. Temperature: $25.0^{\circ} \mathrm{C}$.

fact electromotivity [26] of the cell composed of nonporous polycrystalline hematite electrode and the reference electrode and is a sum of all potential differences in the circuit. The only potential drop which depends on the $\mathrm{pH}$ is the surface potential. Consequently, in order to evaluate surface potentials $\Psi_{0}$ from the electrode potentials $E$ one should locate the point of zero potential $\mathrm{pH}_{\mathrm{pzp}}$. Based on ideas of Zarzycki et al. $[25,27]$ the mean values of electrode potentials of upper (alkalimetric) and lower (acidimetric) curves at certain $\mathrm{pH}$ were taken as the equilibrium values. The appearance of the saddle point of equilibrium function enabled the determination of the point of zero potential $\left(\mathrm{pH}_{\mathrm{pzp}}\right)$. A "symmetry" of acidic (a) and basic (b) branches were taken as criteria: $\Psi_{0, \mathrm{a}}(\Delta \mathrm{pH})=-\Psi_{0, \mathrm{~b}}(\Delta \mathrm{pH})$, where $\Delta \mathrm{pH}=\left|\mathrm{pH}-\mathrm{pH}_{\mathrm{pzp}}\right|$ and $\Psi_{0, \mathrm{a}}$ and $\Psi_{0, \mathrm{~b}}$ are surface potentials corresponding to acidic and basic branches at the same $\Delta \mathrm{pH}$ value. The point of zero potential as obtained by this method was found at $\mathrm{pH}_{\text {pzp }}=7.0$. Using this value, the equilibrium values of surface potentials were calculated and presented in Figure 5 by thick gray band. The original method for the determination of the point of zero potential proposed by Zarzycki et al. $[25,27]$ was also applied. The division of hysteresis loop in two parts having the same area resulted in $\mathrm{pH}_{\text {pzp }} \approx 6.7$. The deviation of the $\mathrm{pH}_{\text {pzp }}$ value obtained from hysteresis loop may be attributed to the difference in mechanisms of charging in the acidic and in the basic region. As it will be shown latter, the "symmetry" of acidic (a) and basic (b) branches produces a reliable point of zero potential, since this approach is in accordance with the surface complexation model. The results are presented in Figure 5 showing that ultrasound causes faster equilibration, especially in the acidic region.

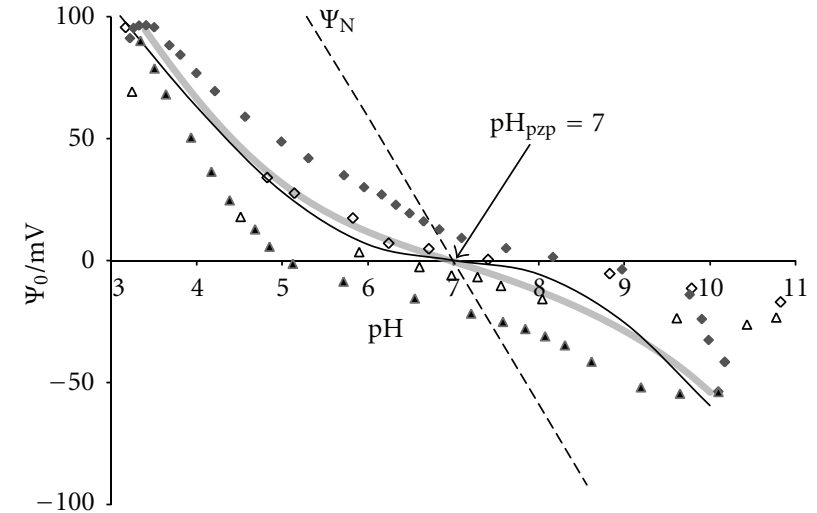

FIGURE 5: Surface potential of polycrystalline hematite in aqueous $\mathrm{NaClO}_{4}$ solution $\left(c=10^{-3} \mathrm{~mol} \mathrm{dm}{ }^{-3}\right)$ at $25.0^{\circ} \mathrm{C}$. Nonequilibrium data are taken from Figure $3(\boldsymbol{\bullet}, \boldsymbol{\Delta})$. Data for partially equilibrated systems (ultrasound) are taken from Figure $4(\diamond, \triangle)$. Evaluated equilibrium values are denoted with the thick gray band. The solid line was calculated on the basis of the surface complexation model, as demonstrated in the next paragraph. The Nernstian potential $\Psi_{\mathrm{N}}$ is denoted with the dashed line.

\section{Theoretical Interpretation}

Interfacial equilibrium parameters, that is, thermodynamic equilibrium constants of surface reactions, could be obtained from surface potential data. The common surface complexation model (SCM) was applied. There are two possible mechanisms of the interactions of $\mathrm{H}^{+}$ions with surface sites. Nonlinearity and appearance of saddle point, of the surface potential function (Figure 5), disagree with a simpler single step protonation (1-pK) assumption [28-30]. Therefore, double-step protonation (2-pK) concept should be applied $[4,31,32]$. According to this concept, the ionic equilibrium at the hematite aqueous interface is described by the following surface reactions and corresponding thermodynamic equilibrium constants, $K_{1}^{\circ}$ and $K_{2}^{\circ}$ :

$$
\begin{gathered}
\equiv \mathrm{FeO}^{-}+\mathrm{H}^{+} \longrightarrow \equiv \mathrm{FeOH} ; \quad \text { st step; } K_{1}^{\circ}, \\
\equiv \mathrm{FeOH}+\mathrm{H}^{+} \longrightarrow \equiv \mathrm{FeOH}_{2}^{+} ; \quad \text { 2nd step; } K_{2}^{\circ}, \\
K_{1}^{\circ}=\exp \left(\frac{\Psi_{0} F}{R T}\right) \cdot \frac{\{\equiv \mathrm{FeOH}\}}{\left\{\equiv \mathrm{FeO}^{-}\right\} \cdot a_{\mathrm{H}^{+}}}, \\
K_{2}^{\circ}=\exp \left(\frac{\Psi_{0} F}{R T}\right) \cdot \frac{\left\{\equiv \mathrm{FeOH}_{2}^{+}\right\}}{\{\equiv \mathrm{FeOH}\} \cdot a_{\mathrm{H}^{+}}},
\end{gathered}
$$

where $\Psi_{0}$ is the surface potential, that is the electrostatic potential affecting the state of charged surface groups $\equiv$ $\mathrm{FeOH}_{2}^{+}$and $\equiv \mathrm{FeO}^{-}$. Surface concentrations (amount per surface area) are denoted with curly braces, while other symbols have their usual meaning.

Note that the first step of protonation is in fact deprotonation of amphoteric $\equiv \mathrm{FeOH}$ surface sites but in the opposite direction.

The surface charge is reduced by association of anions $\mathrm{A}^{-}$ and cations $\mathrm{C}^{+}$with oppositely charged surface groups by

$$
\begin{aligned}
& \equiv \mathrm{FeOH}_{2}^{+}+\mathrm{A}^{-} \longrightarrow \equiv \mathrm{FeOH}_{2}^{+} \cdot \mathrm{A}^{-} ; K_{\mathrm{A}}^{\circ}, \\
& \equiv \mathrm{FeO}^{-}+\mathrm{Na}^{+} \longrightarrow \equiv \mathrm{FeO}^{-} \cdot \mathrm{Na}^{+} ; K_{\mathrm{C}}^{\circ} .
\end{aligned}
$$


The corresponding thermodynamic equilibrium constants $K_{\mathrm{A}}^{\circ}$ and $K_{\mathrm{C}}^{\circ}$ are

$$
\begin{gathered}
K_{\mathrm{A}}^{\circ}=\exp \left(-\frac{\Psi_{\beta} F}{R T}\right) \cdot \frac{\left\{\equiv \mathrm{FeOH}_{2}^{+} \cdot \mathrm{A}^{-}\right\}}{\left\{\equiv \mathrm{FeOH}_{2}^{+}\right\} \cdot a_{\mathrm{A}^{-}}}, \\
K_{\mathrm{C}}^{\circ}=\exp \left(\frac{\Psi_{\beta} F}{R T}\right) \cdot \frac{\left\{\equiv \mathrm{FeO}^{-} \cdot \mathrm{C}^{+}\right\}}{\left\{\equiv \mathrm{FeO}^{-}\right\} \cdot a_{\mathrm{C}^{+}}},
\end{gathered}
$$

where $\Psi_{\beta}$ is the electrostatic potential affecting the state of associated counterions, that is, the potential of the $\beta$-plane in which the centers of associated counterions are located.

Total density of surface sites is given by

$$
\begin{aligned}
\Gamma_{\text {tot }}= & \{\equiv \mathrm{FeOH}\}+\left\{\equiv \mathrm{FeOH}_{2}^{+}\right\}+\left\{\equiv \mathrm{FeOH}_{2}^{+} \cdot \mathrm{A}^{-}\right\} \\
& +\left\{\equiv \mathrm{FeO}^{-}\right\}+\left\{\equiv \mathrm{FeO}^{-} \cdot \mathrm{C}^{+}\right\} .
\end{aligned}
$$

Surface charge density of the 0 -plane is given by

$$
\begin{array}{r}
\sigma_{0}=F \cdot\left(\left\{\equiv \mathrm{FeOH}_{2}^{+}\right\}+\left\{\equiv \mathrm{FeOH}_{2}^{+} \cdot \mathrm{A}^{-}\right\}\right. \\
\left.-\left\{\equiv \mathrm{FeO}^{-}\right\}-\left\{\equiv \mathrm{FeO}^{-} \cdot \mathrm{C}^{+}\right\}\right),
\end{array}
$$

and of the $\beta$-plane

$$
\sigma_{\beta}=F \cdot\left(\left\{\equiv \mathrm{FeO}^{-} \cdot \mathrm{C}^{+}\right\}-\left\{\equiv \mathrm{FeOH}_{2}^{+} \cdot \mathrm{A}^{-}\right\}\right) .
$$

The effective (net) surface charge density $\sigma_{\mathrm{s}}$ is equal in magnitude and opposite in sign to the surface charge density of the diffuse layer $\sigma_{\mathrm{d}}$

$$
\sigma_{\mathrm{s}}=-\sigma_{\mathrm{d}}=\sigma_{0}+\sigma_{\beta}=F \cdot\left(\left\{\equiv \mathrm{FeOH}_{2}^{+}\right\}-\left\{\equiv \mathrm{FeO}^{-}\right\}\right) .
$$

The relationships between surface charge densities and interfacial electrostatic potentials are often based on the concept of condensers of constant capacitance $C$ (expressed per surface area) as

$$
C=\frac{\sigma_{0}}{\Psi_{0}-\Psi_{\beta}}
$$

and by the Gouy-Chapman theory for flat surfaces

$$
\sigma_{\mathrm{d}}=-\sigma_{\mathrm{s}}=-\sqrt{8 R T \varepsilon I_{c}} \sinh \left(-\frac{F \Psi_{\mathrm{d}}}{R T}\right),
$$

where $\varepsilon\left(\varepsilon=\varepsilon_{0} \cdot \varepsilon_{\mathrm{r}}\right)$ is permittivity. $I_{c}$ is the ionic strength determined by the concentrations $(c)$ and the respective charge numbers $(z)$ of all ions present in the system (bulk solution) according to

$$
I_{c}=\frac{1}{2} \sum_{i} c_{i} \cdot z_{i}^{2}
$$

Equations (1)-(13) were used to evaluate protonation equilibrium constants $K_{1}^{\circ}$ and $K_{2}^{\circ}$ from the equilibrium values of surface potentials (Figure 5). Calculations were performed for ionic strength $I_{c}=10^{-3} \mathrm{~mol} \mathrm{dm}^{-3} ; \mathrm{T}=298 \mathrm{~K}$. Parameters used in calculations correspond to a typical metal oxide aqueous dispersion: relative permittivity of water $\varepsilon_{\mathrm{r}}\left(\mathrm{H}_{2} \mathrm{O}\right)=78.54$, capacitance of the inner Helmholtz layer $C=1.5 \mathrm{~F} \mathrm{~m}^{-2}$, and counterion association equilibrium constants $K_{\mathrm{A}}^{\circ}=K_{\mathrm{C}}^{\circ}=50$.

In the absence of specific adsorption, at the point of zero potential $\mathrm{pH}_{\mathrm{pzp}}: \Psi_{0}=0$ and $\left\{\equiv \mathrm{FeO}^{-}\right\}=\left\{\equiv \mathrm{FeOH}_{2}^{+}\right\}$, according to (3) and (4) protonation equilibrium constants are related to $\mathrm{pH}_{\mathrm{pzp}}$ by

$$
\frac{1}{2} \lg K_{1}^{\circ} K_{2}^{\circ}=\mathrm{pH}_{\mathrm{pzp}} .
$$

Equation (14) enables the evaluation of the product of equilibrium constants, while their ratio is determined by the nonlinearity of surface potential, that is, by the appearance of saddle like function

$$
\Delta \mathrm{p} K=\lg \left(\frac{K_{2}^{\circ}}{K_{1}^{\circ}}\right) .
$$

From the best fit, the thermodynamic equilibrium constant of the protonation of negative surface groups is $\lg K_{1}^{\circ}=$ 11.3 , while the thermodynamic equilibrium constant of the protonation of neutral surface groups is $\lg K_{2}^{\circ}=2.8$. The best fit value of the total surface concentration was found to be $\Gamma_{\text {tot }}=1 \cdot 10^{-5} \mathrm{~mol} \mathrm{~m}^{-2}$ (corresponds to 6 sites $/ \mathrm{nm}^{2}$ ). Quality of the fit is demonstrated in Figure 5; solid line was calculated on the basis of obtained values of $K_{1}^{\circ}, K_{2}^{\circ}$ and $\Gamma_{\text {tot }}$. The effect of the choice of values of $C=1.5 \mathrm{~F} \mathrm{~m}^{-2}, K_{\mathrm{A}}^{\circ}$ and $K_{\mathrm{C}}^{\circ}$ will be discussed in the next paragraph.

\section{Discussion}

The polycrystalline hematite mineral enabled the measurements of the surface potential due to its nonporosity. In comparison to single-crystal electrodes, these results are more closely related to colloidal hematite dispersions, since all existing planes are exposed to the liquid medium. In the absence of specific adsorption, and in the symmetric case (same affinities of anions and cations for association with oppositely charged groups) and at low ionic strength, point of zero potential $\mathrm{pH}_{\mathrm{pzp}}$ coincides with the electroneutrality point $\mathrm{pH}_{\mathrm{eln}}$ determined by thermodynamic interfacial equilibrium constants, electrokinetic isoelectric point $\mathrm{pH}_{\text {iep }}$, and with the point of zero charge $\mathrm{pH}_{\mathrm{pzc}}$, as obtained by mass titration or potentiometric titrations of the suspensions [4, 33]. The point of zero potential as determined in this study $\left(\mathrm{pH}_{\mathrm{pzp}}=7\right)$ lies within the values found in the literature for colloid particles and polycrystalline powder $[34,35]$.

Absolute and equilibrium values of the surface potential were obtained from the hysteresis loop. The point of zero potential as determined by the method proposed by Zarzycki $[25,28]$ that is from the surface area of hysteresis loop agrees fairly with the value obtained by considering "symmetry" of the saddle like surface potential function that is by the method based on the surface complexation model. The interpretation yielded thermodynamic equilibrium constants for 
the first and the second step of protonation of the surface sites. The advantage of interpretation of surface potential data, with respect to commonly used surface charge data, is that the surface potential is not sensitive to the choice of counterion association equilibrium constants. The values between 10 and 1000 produce a satisfying fit. Consequently, surface potential data cannot be used for evaluation of these constants, but on the other hand, the evaluated protonation equilibrium constants are more reliable $\left(\lg K_{1}^{\circ}=11.3\right.$ and $\left.\lg K_{2}^{\circ}=2.8\right)$. The similar applies to the choice of the electrical capacitance $C$, all values between $1 \mathrm{~F} \mathrm{~m}^{-2}$ and $2 \mathrm{~F} \mathrm{~m}^{-2}$ fit the experimental surface potential data. However, the total density of surface sites could be evaluated. The best fit value of $\Gamma_{\text {tot }}=1 \cdot 10^{-5} \mathrm{~mol} \mathrm{~m}^{-2}$ agrees with the commonly used value as deduced from the surface structure.

The main achievement of surface potential measurements lies in the fact that these data may distinguish between single $(1-\mathrm{pK})$ and double $(2-\mathrm{pK})$ protonation mechanisms. Single protonation would result in the smooth, almost linear dependency on $\mathrm{pH}$, but cannot interpret a saddle-like shape. On the contrary, most of the surface charge data could be successfully interpreted by both-single and double-step assumptions. The appearance of the saddle point of the surface potential suggest the double step mechanism; the first step is responsible for surface charging in the basic region, but the second step determines the surface charge in the acidic region. The high $\Delta \mathrm{p} K$ value $(\Delta \mathrm{p} K=8.5)$ indicates the broad saddle region.

In conclusion, this paper demonstrates the power of recently introduced surface potential measurements by using the nonporous metal oxide electrodes.

\section{Acknowledgments}

This research was supported by the Ministry of Science, Education, and Sports of the Republic of Croatia (Project no. 119-1191342-2961). The authors are grateful to Dr. Stjepko Krehula for the photography of polycrystalline hematite sample.

\section{References}

[1] J. Lützenkirchen, "On derivatives of surface charge curves of minerals," Journal of Colloid and Interface Science, vol. 290, no. 2, pp. 489-497, 2005.

[2] W. Stumm, C. P. Huang, S. R. Jenkins et al., "Specific chemical interaction affecting the stability of dispersed systems," Croatica Chemica Acta, vol. 42, pp. 223-245, 1970.

[3] D. E. Yates, S. Levine, and T. W. Healy, "Site-binding model of the electrical double layer at the oxide/water interface," Journal of the Chemical Society, Faraday Transactions I, vol. 70, pp. 1807-1818, 1974.

[4] J. Lützenkirchen, Ed., Surface Complexation Modelling, Interface Science and Technology, Elsevier, 2006.

[5] N. Kallay, Z. Dojnović, and A. Čop, "Surface potential at the hematite-water interface," Journal of Colloid and Interface Science, vol. 286, no. 2, pp. 610-614, 2005.

[6] S. V. Yanina and K. M. Rosso, "Linked reactivity at mineralwater interfaces through bulk crystal conduction," Science, vol. 320, no. 5873, pp. 218-222, 2008.
[7] L. Bousse, N. F. de Rooij, and P. Bergveld, "The influence of counter-ion adsorption on the $\psi 0 / \mathrm{pH}$ characteristics of insulator surfaces," Surface Science, vol. 135, no. 1-3, pp. 479496, 1983.

[8] C. Cichos and T. Geidel, "Contribution to direct measurement of double-layer potential at the oxide/electrolyte interface," Colloid \& Polymer Science, vol. 261, no. 11, pp. 947-953, 1983.

[9] N. H. G. Penners, L. K. Koopal, and J. Lyklema, "Interfacial electrochemistry of haematite $(\alpha-\mathrm{Fe} 2 \mathrm{O} 3)$ : homodisperse and heterodisperse sols," Colloids and Surfaces A, vol. 21, pp. 457468, 1986.

[10] T. Preočanin, A. Čop, and N. Kallay, "Surface potential of hematite in aqueous electrolyte solution: hysteresis and equilibration at the interface," Journal of Colloid and Interface Science, vol. 299, no. 2, pp. 772-776, 2006.

[11] N. Kallay, T. Preočanin, J. Marković, and D. Kovačević, "Adsorption of organic acids on metal oxides: application of the surface potential measurements," Colloids and Surfaces A, vol. 306, no. 1-3, pp. 40-48, 2007.

[12] T. Preočanin, Ž. Majić, D. Kovačević, and N. Kallay, "Adsorption of oxalic acid onto hematite: application of surface potential measurements," Adsorption Science and Technology, vol. 25, no. 6, pp. 429-437, 2007.

[13] T. Preočanin and N. Kallay, "Effect of electrolyte on surface potential of haematite in aqueous electrolyte solutions," Surface Engineering, vol. 24, no. 4, pp. 253-258, 2008.

[14] T. Preočanin, W. Janusz, and N. Kallay, "Evaluation of equilibrium parameters of the anatase/aqueous electrolyte solution interface by introducing surface potential data," Colloids and Surfaces A, vol. 297, no. 1-3, pp. 30-37, 2007.

[15] T. Preočanin, A. Selmani, D. Mazur, and N. Kallay, "The effect of electrolytes on the surface potential at the rutile/aqueous interface," Applied Surface Science, vol. 256, no. 17, pp. 54125415, 2010.

[16] T. Preočanin, M. Tuksar, and N. Kallay, "Mechanism of charging of the pyrite/aqueous interface as deduced from the surface potential measurements," Applied Surface Science, vol. 253, no. 13, pp. 5797-5801, 2007.

[17] J. Lützenkirchen, R. Zimmermann, T. Preočanin et al., "An attempt to explain bimodal behaviour of the sapphire c-plane electrolyte interface," Advances in Colloid and Interface Science, vol. 157, no. 1-2, pp. 61-74, 2010.

[18] N. Kallay and D. Cakara, "Reversible charging of the ice-water interface: I. Measurement of the surface potential," Journal of Colloid and Interface Science, vol. 232, no. 1, pp. 81-85, 2000.

[19] N. Kallay, A. Čop, E. Chibowski, and L. Holysz, "Reversible charging of the ice-water interface: II. Estimation of equilibrium parameters," Journal of Colloid and Interface Science, vol. 259, no. 1, pp. 89-96, 2003.

[20] N. Kallay, T. Preočanin, and F. Šupljika, "Measurement of surface potential at silver chloride aqueous interface with single-crystal AgCl electrode," Journal of Colloid and Interface Science, vol. 327, no. 2, pp. 384-387, 2008.

[21] T. Preočanin, F. Šupljika, and N. Kallay, "Evaluation of interfacial equilibrium constants from surface potential data: silver chloride aqueous interface," Journal of Colloid and Interface Science, vol. 337, no. 2, pp. 501-507, 2009.

[22] T. Preočanin, F. Šupljika, and N. Kallay, "Charging of silver bromide aqueous interface: evaluation of interfacial equilibrium constants from surface potential data," Journal of Colloid and Interface Science, vol. 346, no. 1, pp. 222-225, 2010.

[23] T. Preočanin, F. Šupljika, and N. Kallay, "Charging of silver bromide aqueous interface: evaluation of enthalpy and entropy of interfacial reactions from surface potential data," 
Journal of Colloid and Interface Science, vol. 354, no. 1, pp. 318321, 2011.

[24] N. Kallay and T. Preočanin, "Measurement of the surface potential of individual crystal planes of hematite," Journal of Colloid and Interface Science, vol. 318, no. 2, pp. 290-295, 2008.

[25] P. Zarzycki, K. M. Rosso, S. Chatman, T. Preočanin, N. Kallay, and W. Piasecki, "Theory, experiment and computer simulation of the electrostatic potential at crystal/electrolyte interfaces," Croatica Chemica Acta, vol. 83, no. 4, pp. 457-474, 2010.

[26] V. Simeon, "Electromotivity to replace electromotive force?" Chemistry International, vol. 26, no. 6, p. 12, 2004.

[27] P. Zarzycki, T. Preočanin, S. Chatman, and K. M. Rosso, "The electrostatic potentional of specific mineral faces," Langmuir, vol. 27, no. 13, pp. 7986-7990, 2011.

[28] W. H. van Riemsdijk, G. H. Bolt, L. K. Koopal, and J. Blaakmeer, "Electrolyte adsorption on heterogeneous surfaces: adsorption models," Journal of Colloid and Interface Science, vol. 109, no. 1, pp. 219-228, 1986.

[29] R. H. Yoon, T. Salman, and G. Donnay, "Predicting points of zero charge of oxides and hydroxides," Journal of Colloid and Interface Science, vol. 70, no. 3, pp. 483-493, 1979.

[30] T. Hiemstra, P. Venema, and W. H. van Riemsdijk, "Intrinsic proton affinity of reactive surface groups of metal (Hydr)oxides: the bond valence principle," Journal of Colloid and Interface Science, vol. 184, no. 2, pp. 680-692, 1996.

[31] J. A. Davis, R. O. James, and J. O. Leckie, "Surface ionization and complexation at the oxide/water interface. I. Computation of electrical double layer properties in simple electrolytes," Journal of Colloid and Interface Science, vol. 63, no. 3, pp. 480499, 1978.

[32] W. Rudziński, R. Charmas, W. Piasecki et al., "Calorimetric effects of simple ion adsorption at oxide/electrolyte interface: titration experiments and their theoretical analysis based on 2-pK charging mechanism and on the triple layer model," Colloids and Surfaces A, vol. 137, pp. 57-68, 1998.

[33] G. Sposito, "On points of zero charge," Environmental Science and Technology, vol. 32, no. 19, pp. 2815-2819, 1998.

[34] L. Cromières, V. Moulin, B. Fourest, and E. Giffaut, "Physicochemical characterization of the colloidal hematite/water interface: experimentation and modelling," Colloids and Surfaces A, vol. 202, no. 1, pp. 101-115, 2002.

[35] M. Kosmulski, "pH-dependent surface charging and points of zero charge. IV. Update and new approach," Journal of Colloid and Interface Science, vol. 337, no. 2, pp. 439-448, 2009. 


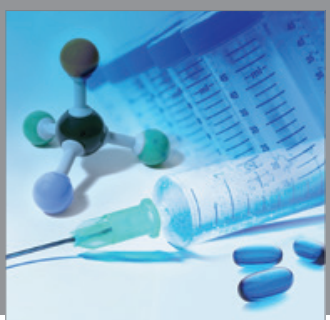

International Journal of

Medicinal Chemistry

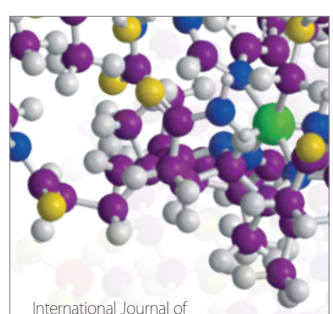

Carbohydrate Chemistry

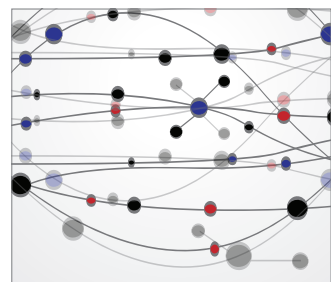

The Scientific World Journal
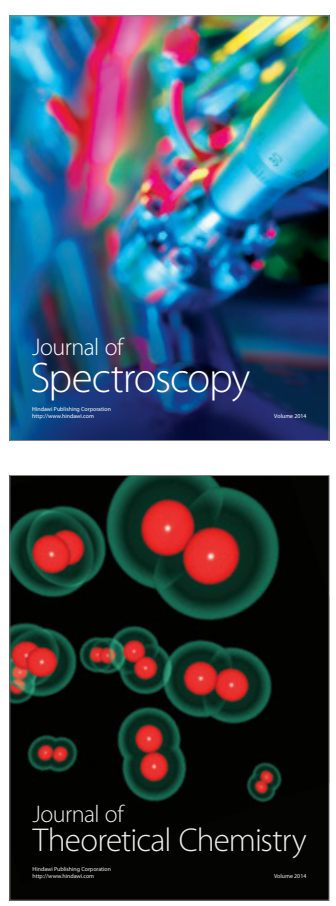
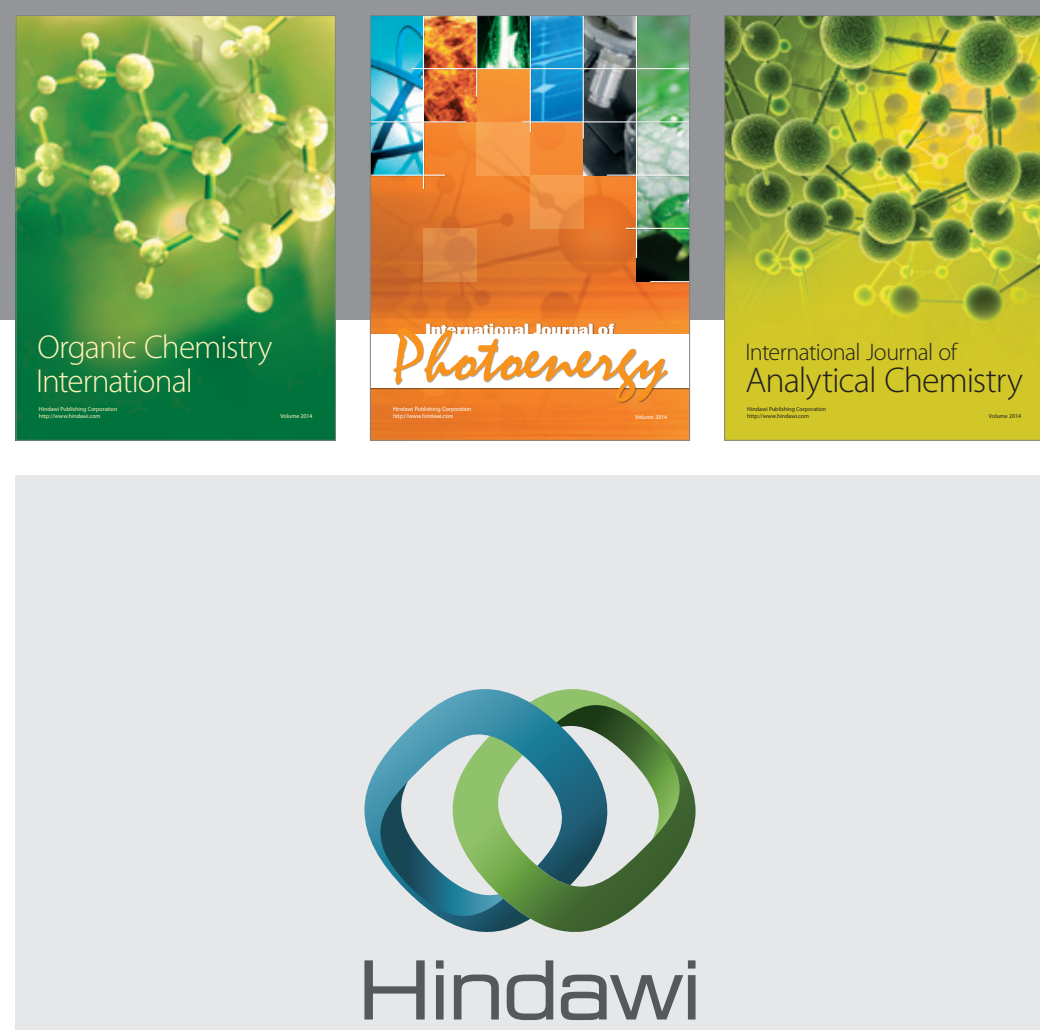

Submit your manuscripts at

http://www.hindawi.com
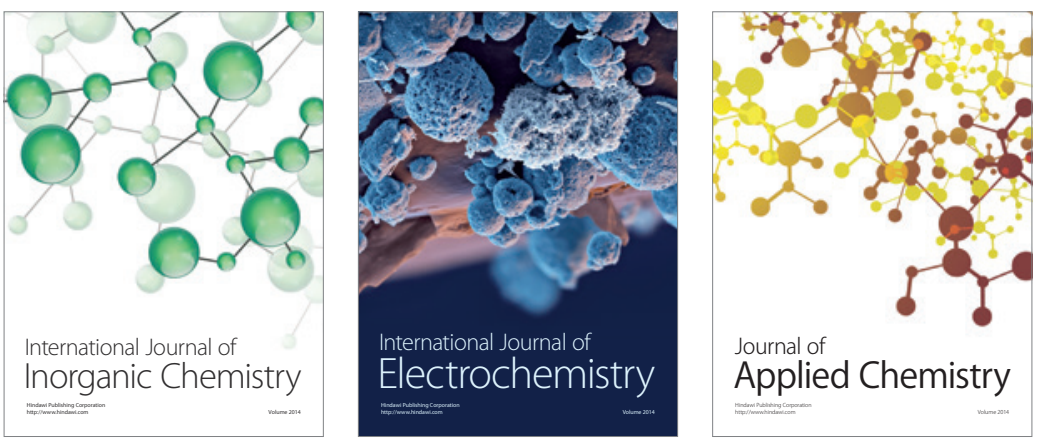

Journal of

Applied Chemistry
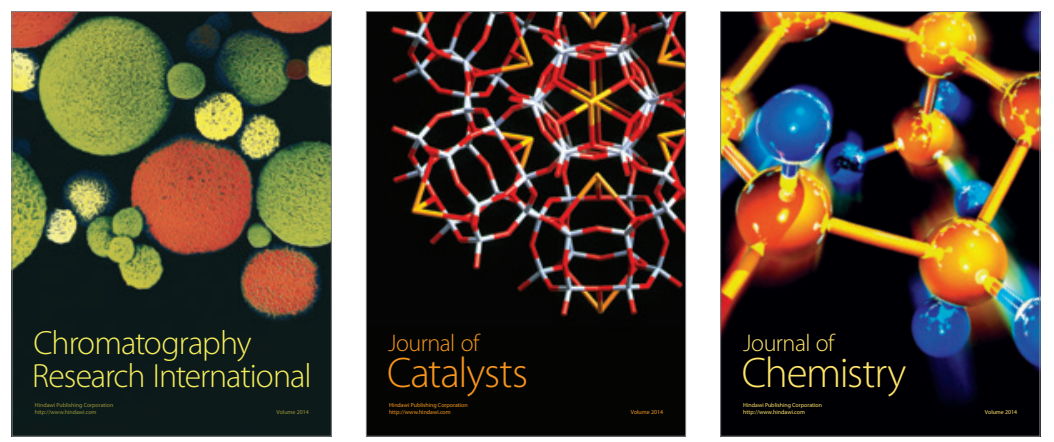
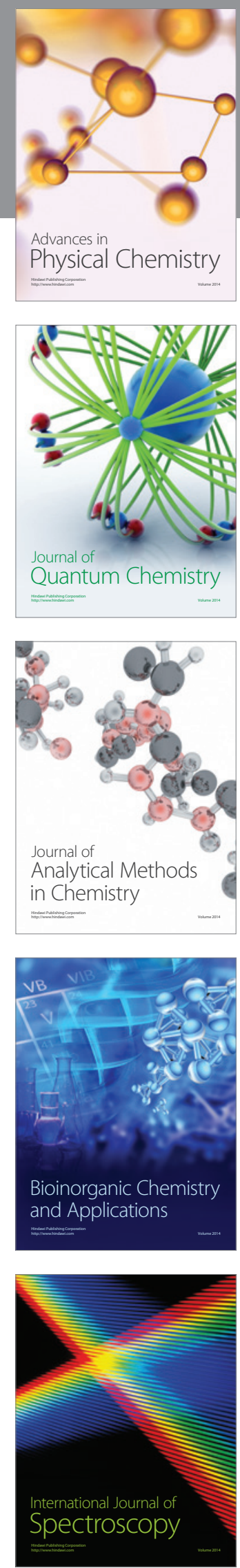